\title{
ARTICLE
}

\section{Response of the imaging plates to the electron beams from a linear accelerator at ultra-low intensity}

\author{
Hiromoto Shimomura ${ }^{*}$, Ryoichi Taniguchi, Shuichi Okuda, \\ Hiroyuki Miyamaru, Hiroto Matsuura and Takao Kojima \\ Radiation Research Center, Osaka Prefecture University, Gakuen- cho 1-2, Nakaku, Sakai, Osaka 599-8570, Japan
}

\begin{abstract}
The response of the imaging plate (IP) as a two-dimensional irradiation dose monitor of high-energy electrons has been investigated. The Co-60 $\gamma$-ray irradiation facilities and the $16 \mathrm{MeV}$ S-band electron linear accelerator (linac) in Radiation Research Center, Osaka Prefecture University have been used for the experiments. From the results of the irradiation experiments with $\gamma$-rays uniform response of IP over the plate and linear response to the exposure have been obtained. Thermoluminescence dosimeter has been used to determine the exposure. The ultra-low intensity electron beams developed have been used. The results of the irradiation experiments with the narrow electron beams from the linac at an energy of $8 \mathrm{MeV}$ have shown the linear response of IP to the total electron charge for irradiation. By the broad beam an IP image of a model sample has been obtained showing the characteristic scattering behavior of high-energy electrons. These results have shown the applicability of IP to monitoring two-dimensional dose distribution of electrons.
\end{abstract}

Keywords: imaging plates; two-dimensional dose distributions; ultra-low intensity electron beams; electron linear accelerator; gamma-rays; thermoluminescence dosimeter

\section{Introduction}

Imaging plates (IP) have been widely used for monitoring the two-dimensional images of ionizing radiation at relatively high sensitivity. The researches to apply the IP to monitoring X-ray exposure distribution have been conducted in the field of medical applications [1]. The study of the response of IP to high-energy electrons is important in the investigation of the shielding properties of inhomogeneous matters and the evaluation of the dose distribution of electrons especially for medical applications. In order to investigate the response of such highly sensitive radiation dosimeters to energetic electrons changing the electron energy, different kinds of radioisotopes as beta-ray sources have been generally used. In the case of electron beams from a linear accelerator (linac) the energy, the position and the other beam conditions can be precisely changeable. However, the intensity of the electron beam in the ordinary operational conditions is extremely high for such experiments.

The sensitivity of IP to high-energy electron beams from linac was measured at very low beam intensity in order to investigate the property of an electron energy spectrometer by Tanaka et al. [2]. However, the beam conditions were limited and could not be widely controlled. For the detailed investigation of the response

\footnotetext{
*Corresponding author. Email:dw105008@edu.osakafu-u.ac.jp
}

of IP the establishment of the irradiation field of the ultra-low intensity electron beams is necessary.

Recently, we succeed in generating ultra-low intensity electron beams stably by using a $16 \mathrm{MeV}$ S-band linac in Radiation Research Center, Osaka Prefecture University (OPU). This was achieved by the development of the methods for controlling the components of the electron linac and for measuring the low intensity beams [3]. The minimum electron charge in a beam macro pulse obtained in relatively stable beam conditions was about $1 \mathrm{fC}$. The beams have been applied to investigating the response of highly sensitive dosimeters.

In the present work the response of IP to the high-energy electron beams has been investigated by using the ultra-low intensity electron beams in OPU.

\section{Properties of the imaging plate and the digital imaging system}

In the present experiments the commercial product of IP for X-ray radiography (CR SR-VI, Fuji Film) and the readout unit (AC-7/ST, Fuji Film) were used. The thickness of the sensor layer is $120 \mu \mathrm{m}$, the dimensions of the IP $20 \mathrm{~cm} \times 25 \mathrm{~cm}$ and the pixel size $0.1 \mathrm{~mm} \times 0.1$ $\mathrm{mm}$.

In the irradiation experiments the IP was installed in a cassette (type CC). The absorbed dose distribution is 
given from the position and the intensity of PSL on IP [1, $4,5]$.

The exposure of the $\gamma$-rays or the irradiation dose of the electron beams in the present experiments was sufficiently low so that no afterimage was left behind on the IP. The IP was used repeatedly after the readout procedure. By using an IP for investigating the response in the experiments the results were not affected by the difference between the plates.

The main properties of IP as the two-dimensional dosimeter are the uniformity in the sensitivity over the plate, the reproducibility in the readout procedure of the IP, and the fading after irradiation with ionizing radiation. The fading depends on the type of IP and surrounding temperature. In the present experiments the fading was evaluated in each measurement.

By the digital imaging system an image is given along with some indexes. The intensity of the output PSL signal of IP which is corresponding to the absorbed dose was evaluated from the indexes.

\section{Irradiation experiments}

\subsection{Irradiation with Co-60 $\gamma$-rays}

The $\gamma$-ray irradiation experiments were performed to investigate the basic properties of the IP used in the present work, such as the response of IP to the exposure and the uniformity of IP over the plate. The experimental conditions can be easily achieved by using the $\gamma$-ray irradiation facilities in OPU.

Figure 1 schematically shows the configurations of the irradiation experiment with a $3.7 \mathrm{GBq}$ Co-60 $\gamma$-ray source. The irradiation device for non-destructive inspection consists of a lead container, a source guiding tube, and a remote control conduit. The source was introduced to the head of the guide tube via a manual crank-out mechanism. Thermoluminescence dosimeter (TLD, UD-802PQ, Panasonic) was used as a local exposure monitor. The TLD element of number 3 for $\gamma$-, $\mathrm{X}-$, and beta-rays was used in this study.

In the irradiation experiment with $\gamma$-rays the TLD and the IP were placed at $400 \mathrm{~mm}$ from the source and the IP was normally irradiated. The deviation of the exposure from uniform irradiation over the plate due to the configurations shown in Figure 1 was taken into account in the uniformity evaluation for the IP sensitivity. The temperature in the experiment was $20^{\circ} \mathrm{C}$. The time from the $\gamma$-ray irradiation and the readout of IP was about 15 minutes. The fading of IP during the irradiation was evaluated in the present results.

\subsection{Irradiation with ultra-low intensity linac electron beams}

The OPU S-band linac and the beam-transport system is schematically shown in Figure 2. The ultra-low intensity electron beams from the linac were used in this experiment.

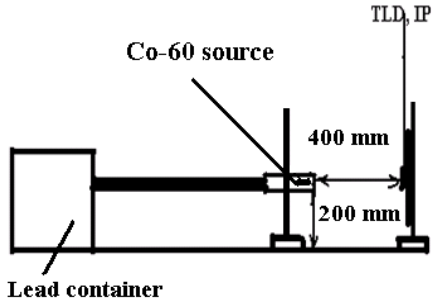

Figure 1. Schematic diagram showing the configurations of the irradiation experiment with Co-60 $\gamma$-rays.

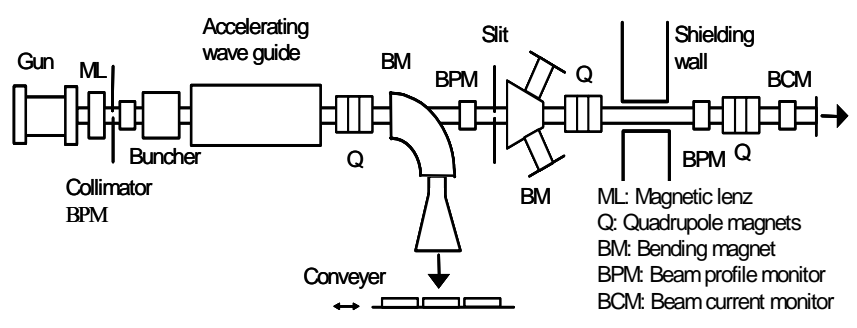

Figure 2. Schematic diagram of the OPU S-band linac and the beam-transport system.

In order to generate the ultra-low intensity beams operational conditions of the linac components were controlled as follows: the injection current from the electron gun was minimized by decreasing the heater current of the thermionic gun cathode under stable conditions; and the beam pulse width was decreased.

After acceleration the beam was bent to an underground irradiation room, where the energy spectrum of the beam was measured. Monitoring the electron beam in this room the operational conditions of the linac were optimized to obtain the stable beam and to determine the peak beam energy. The beams were then transported to the straight direction. The intensity of the accelerated beam was further decreased by using water cooled beam slits, whose beam attenuation factor measured in a typical case was about $1 / 350$. The beam was transported through a concrete shielding wall in order to avoid the Bremsstrahlung X-ray background in the accelerator room, and was used in the other irradiation room for irradiation experiments. The minimum electron charge in an electron beam so far obtained in a relatively stable conditions was about 1 $\mathrm{fC} /$ pulse. In the present experiments the pulse width of the electron beam was $4 \mu \mathrm{s}$, the beam energy about 8 $\mathrm{MeV}$ and the electron charge about $10 \mathrm{pC} /$ pulse.

The real-time profile of the focused electron beam was observed by using a $\mathrm{ZnCdS}$ scintillator and a CCD camera at relatively high charges in the range of $\mathrm{nC}$. This monitoring system was used in the procedure of beam conditioning. For the lower charge the profile is possibly monitored by using IP.

Figure 3 schematically shows the configurations of the irradiation experiment with ultra-low linac electron beams. 
The beam was focused on the vacuum Ti window 50 $\mu \mathrm{m}$ thick with focusing magnets. The IP placed at 400 $\mathrm{mm}$ from the window was normally irradiated with the beam at a temperature of $20{ }^{\circ} \mathrm{C}$. The total charge of electrons in the irradiation was changed by changing the number of the electron beam pulse. After about 15 minutes from the irradiation the IP was read out. The contribution of the fading of IP during the irradiation to the result was evaluated to be sufficient small. In the experiments the influence of the Bremsstrahlung X-rays from the electron beams and background X-rays were negligible compared with the irradiation dose of the electrons.

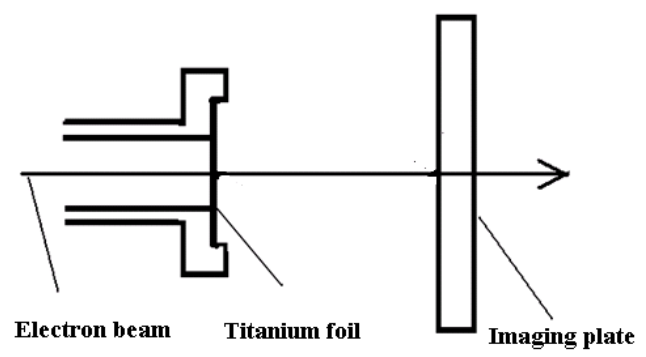

Figure 3. Schematic diagram of the experimental setup of the ultra-low intensity linac electron beam irradiation.

\section{Results and discussion}

\subsection{Results of the irradiation experiment with $\gamma$-rays}

The basic properties of IP as a two-dimensional dosimeter have been investigated by using $\gamma$-rays. It is difficult to evaluate the absorbed dose in the sensor layer of IP. In the experiments the exposure was evaluated from the absorbed dose measured with TLD. The exposure rate evaluated was $0.039(\mathrm{nC} / \mathrm{kg}) / \mathrm{s}$. In the measurements the same IP and TLD were used repeatedly.

Figure 4 shows the relation between the $\gamma$-ray exposure and the output PSL signal of IP given by the IP index data obtained from the readout procedure. The main causes of the error were in the evaluation of the exposure rate and the deviation from the uniformity of IP image over the plate. The maximum error in the data was about $3 \%$. The uniformity in the output PSL signal of IP over the plate was evaluated for each data points shown in Figure 4. It was found within $1 \%$. In the previous work it was reported to be $1.6 \%$ [1].

In Figure 4 one can see the linear response of IP. These results show the applicability of the IP as the two-dimensional exposure monitor with relatively high accuracy for $\gamma$-rays.

\subsection{Results of the irradiation experiment with ultra-low intensity linac electron beams}

Figure 5 shows the typical IP image of the ultra-low linac electron beam used for investigating the response of IP. The narrow beam was used in this case.

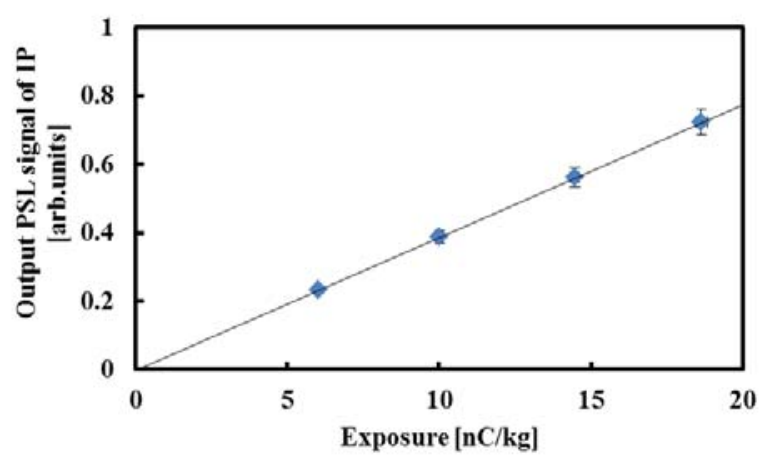

Figure 4. Relation between the $\gamma$-ray exposure and the output PSL signal of IP.

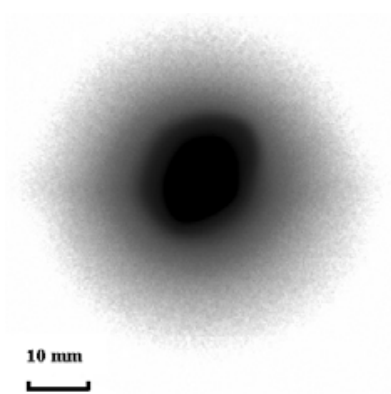

Figure 5. Typical image of the ultra-low intensity linac electron beams.

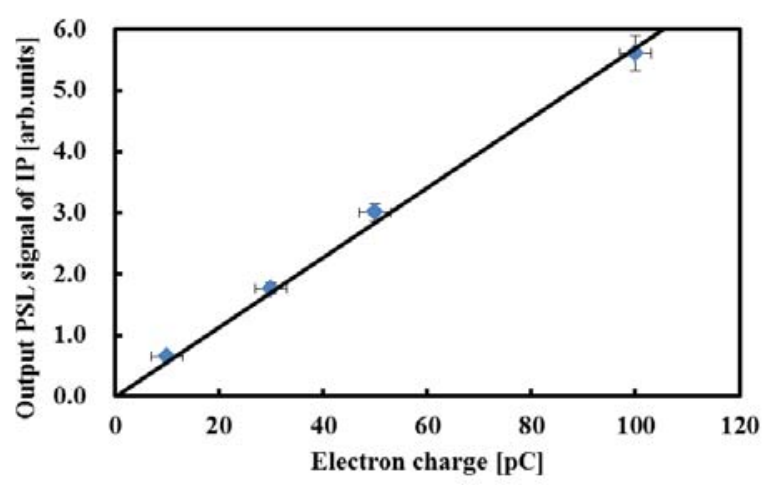

Figure 6. Relation between the electron charge in the irradiated electron beams and the output PSL signal of IP.

Figure 6 shows the relation between the electron charge in the irradiated electron beams and the output PSL signal of IP obtained for one plate. The electron charge was from 10 to $100 \mathrm{pC}$. In this case the PSL signal corresponds to that of the peak value in the beam intensity profile. The error shown in the electron charge was due to that in the measurement with a charge sensitive monitor. In this figure one can see the linear response of IP.

The results of the present work for $\gamma$-rays and electron beams show the applicability of the two-dimensional imaging method with IP to ultra-low linac beams with relatively high accuracy.

For the same absorbed dose the irradiation dose rate in the electron-beam irradiation is extremely high compared with the exposure rate in the $\gamma$-ray irradiation. 
The further experiments to investigate to compare the sensitivities of IP for the different kinds of ionizing radiations and for the different energies of electrons are under preparation. The evaluation of the sensitivities will be made by determining the absorbed dose of IP using TLD.

\subsection{IP image of a model sample}

In order to investigate the basic properties of the radiation shield to high-energy electrons the ultra-low intensity electron beams were applied to obtain the IP image of a model sample. In order to prepare a broad beam for irradiation, an aluminum plate $2 \mathrm{~mm}$ thick was placed as a diffuser outside of the vacuum window in the configurations as shown in Figure 3. The model sample was a circular glass plate with the diameter of $60 \mathrm{~mm}$ and the thickness of $2 \mathrm{~mm}$. The sample was fixed on the front surface of the IP with a thin film tape. The distance between the window and the sample was $400 \mathrm{~mm}$. Figure 7 shows the images of the sample obtained for an electron beam pulse with an electron charge of about 10 $\mathrm{pC}$ at an energy of $8 \mathrm{MeV}$. As shown in this figure the dose of electrons become relatively high locally at the edge of the sample. This phenomenon is attributed to the characteristic scattering behaviors of electrons. Even the edge of the thin film can be observed. The results of the present work suggest the importance in the applications of IP to monitoring the two-dimensional irradiation dose distribution of high-energy electrons.

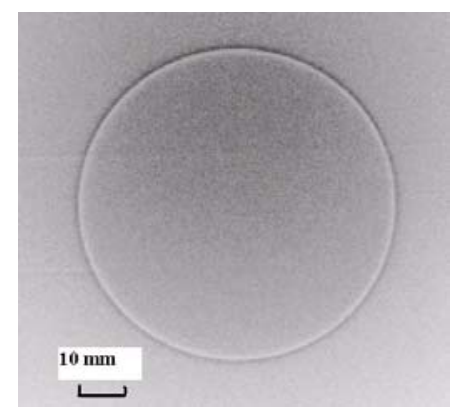

Figure 7. The IP image of the glass plate model sample irradiated with a broad electron beam from a linac.

The possible application of the linac beam is the time-resolved imaging applying the pulse characteristics of the linac beam. The linac pulse duration can be changed from pico second to milli second. Further study using the characteristic irradiation filed is in progress.

\section{Conclusion}

In the present work the response of IP as a two-dimensional electron beam irradiation dose monitor was investigated. The Co-60 $\gamma$-rays irradiation facilities and the $16 \mathrm{MeV}$ S-band electron linac in Radiation Research Center, Osaka Prefecture University were used for the experiments.

In the $\gamma$-ray irradiation experiment the uniformity of the sensitivity of IP over the plate was found to be within $1 \%$. The linear relation between the exposure of $\gamma$-rays and the output PSL signal of IP was obtained. In the experiments of the ultra-low intensity linac electron beam irradiation the energy of the electron beam was 8 $\mathrm{MeV}$ and the electron charge from 10 to $100 \mathrm{pC}$. The linear relation between the electron charge and the output PSL signal of IP was obtained. These results of the present work showed the applicability of the method of the two-dimensional imaging with IP to ultra-low linac beams with relatively high accuracy.

The characteristic IP image of a model sample was obtained by using a broad electron beam according to the scattering behaviors of high-energy electrons. This result suggested the importance in the applications of IP to monitoring the local irradiation dose of electrons and to developing the new imaging method with high-energy electron beams.

\section{Acknowledgements}

This work was supported in part by the program of KEK for supporting accelerator research activities in university.

\section{References}

[1] J. Miyahara, Y. Amemiya and T. Matsushita, Imaging plate, J. Phys. Soc. Jpn. 45 (1990), pp. 398-404. [in Japanese]

[2] K. Tanaka, T. Yabuuchi, T. Takahasi, T. Ikeda and S. Okuda, Calibration of imaging plate for high energy electron spectrometer, Rev. Sci. Instrum. 76, 013507 (2005), pp. 1-5.

[3] R. Taniguchi, T. Kojima and S. Okuda, Ultra-low intensity electron beams of a linear accelerator for irradiation, Radiat. Phys. Chem. 76 (2007), pp. 1779-1782.

[4] K. Takahashi, Progress in science and technology on photostimulable $\mathrm{BaFX}: \mathrm{Eu}^{2+}(\mathrm{X}=\mathrm{Cl}, \mathrm{Br}, \mathrm{I})$ and imaging plates, J. Luminescence 100, 1-4 (2002), pp. 307-315.

[5] J. Shiraishi, D. Tatsumi, H. Huuma, A. Utsunomiya, K. Kusumi and K. Doi, Estimation of patient dose by using a digital imaging system, Jpn. J. Radiological Technol. 57 (2001), pp. 860-867. 